\title{
Rotons in gaseous Bose-Einstein condensates irradiated by a laser
}

\author{
D.H.J. O'Dell ${ }^{1}$, S. Giovanazzi ${ }^{2}$ and G. Kurizki ${ }^{3}$ \\ ${ }^{1}$ Physics and Astronomy Department, University of Sussex, \\ Brighton BN1 9QH, UK \\ ${ }^{2}$ Center for Theoretical Physics and College of Science, Polish Academy of Sciences \\ Aleja Lotników, 32/46, 02-668 Warsaw, Poland \\ ${ }^{3}$ Chemical Physics Department, Weizmann Institute of Science, \\ 76100 Rehovot, Israel
}

September 2, 2018

A gaseous Bose-Einstein condensate (BEC) irradiated by a far off-resonance laser has long-range interatomic correlations caused by laser-induced dipole-dipole interactions. These correlations, which are tunable via the laser intensity and frequency, can produce a 'roton' minimum in the excitation spectrum - behavior reminiscent of the strongly correlated superfluid liquid helium II. PACS: 03.75.Kk, 32.80.Qk, 34.20.Cf, 67.40.Db

According to the celebrated Bijl-Feynman formula [1] for the excitation spectrum of helium II

$$
E(k) \leq \frac{\hbar^{2} k^{2}}{2 m S(k)},
$$

the peculiar "roton" minimum at $k \approx 2 \pi / r_{0}$, where $r_{0}$ is the average atomic separation, is due to a corresponding peak in the static structure factor $S(k) \equiv\left\langle 0\left|\hat{\rho}_{k} \hat{\rho}_{k}^{\dagger}\right| 0\right\rangle / N$. Here $N$ is the number of atoms of mass $m,|0\rangle$ the ground state of the system, and $\hat{\rho}_{k} \equiv \sum_{q} \hat{c}_{q}^{\dagger} \hat{c}_{q+k}$ the density fluctuation operator. $S(k)$ is the Fourier transform of the pair correlation function and hence provides a measure of the degree of pair (2nd order) correlation between the atoms. The existence of strong pair correlations in helium II may at first seem surprising since it remains a liquid even at temperatures approaching absolute zero precisely because of weak interatomic interactions (in combination with a small atomic mass) 22. However, despite their apparent weakness these interactions are very effective because the density of the liquid state is such that the average atomic separation, $r_{0}=4.44 \AA$, is close to the minimum of the attractive interatomic potential well at $3 \AA$.

Contrast this now with an ultra-cold alkali atom gas in which the Bose-Einstein condensed fraction can be very nearly $100 \%$ 4. The interactions in ${ }^{87} \mathrm{Rb}$, for example, are repulsive and characterized by an s-wave scattering length, $a \approx 5.5 \mathrm{~nm}$. This is between one and two orders of magnitude smaller than the average atomic spacing at typical densities. Steinhauer et al [5] recently measured the bulk excitation spectrum of a ${ }^{87} \mathrm{Rb}$ BEC and found excellent agreement with Bogoliubov theory [3] (appropriate for a degenerate almost ideal Bose gas). There was no roton minimum, a consequence of the diluteness with respect to $a$. Indeed, since Eq. (11) becomes an equality within the Bogoliubov theory [1] one sees the pair correlation is small compared to helium II. Significant pair correlation might exist 
in gaseous BECs at the very small scale of $a$, but this is fairly inaccessible in such a delicate system.

A marvellous feature of atoms though, is that their interactions can be manipulated using external fields, allowing us to microscopically engineer the macroscopic properties of a many-body system. Thus the experiment of Inouye et al 6] took advantage of a Feshbach resonance to change the s-wave scattering length using magnetic fields. We have recently proposed the use of off-resonant lasers to induce long-range dipole-dipole interactions whose characteristic length is the laser wavelength. These interactions can cause laser induced self- "gravity" in a BEC, leading to 3-dimensional self-trapping and electrostriction accompanied by unusual excitation spectra [7, as well as "supersolid" structures [8]. The aim here is to explore how the excitation spectrum and, by virtue of (11), the correlations of a gaseous BEC are changed when the interatomic potential is modified via laserinduced dipole-dipole interactions. This task requires a knowledge of the Fourier transform (FT) of the total interatomic potential, so we turn to this first.

Consider a BEC confined by a potential $H_{\text {trap }}=\frac{m}{2} \omega_{r}^{2}\left(x^{2}+y^{2}\right)+\frac{m}{2} \omega_{z}^{2} z^{2}$ into a very elongated cigar shape $\left(\omega_{r} \gg \omega_{z}\right)$, irradiated by a far off-resonance plane-wave laser (Fig. 11 inset). The laser polarization is along the long z-axis of the condensate to suppress collective ("superradiant") Rayleigh scattering 9] or coherent atomic recoil lasing [10 that are forbidden along the direction of polarization. The far off-resonance condition, together with the small extent of the BEC along the laser propagation direction, enables us to treat the electromagnetic field inside the BEC in the Born approximation (field at each point is the sum of the incident plus once-scattered fields). Then the dipole-dipole potential between two atoms of separation $\mathbf{r}$, induced by far-off resonance light of intensity $I$, wave-vector $\mathbf{k}_{\mathrm{L}}=k_{\mathrm{L}} \hat{\mathbf{y}}$ (along the $\mathrm{y}$-axis), and

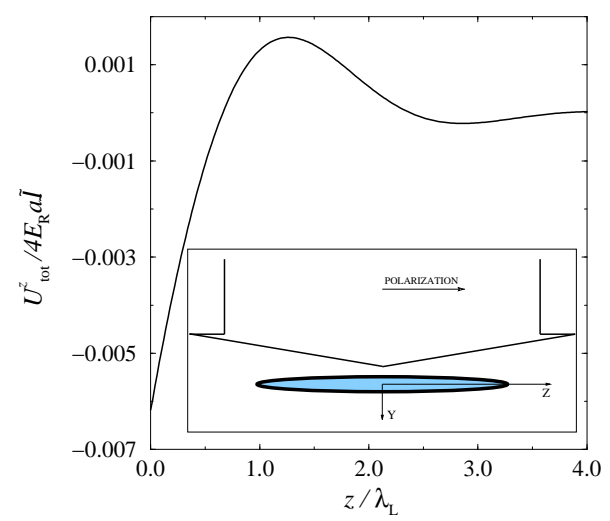

Figure 1: The total (s-wave+dipole-dipole) 1D interatomic potential $U_{\text {tot }}^{z}(z)$ (FT of (6)), for $w_{r}=3.5 \lambda_{\mathrm{L}}$. A repulsive contact term $4 E_{\mathrm{R}} a\left(k_{\mathrm{L}} w_{r}\right)^{-2}(1+4 \mathcal{I} / 3) \delta\left(k_{\mathrm{L}} z\right)$ is not shown. Inset: The laser beam and condensate geometry.

polarization $\hat{\mathbf{e}}=\hat{\mathbf{z}}$ (along the $\mathrm{z}$-axis) is 11 .

$$
U_{\mathrm{dd}}(\mathbf{r})=\frac{I \alpha^{2}(\omega) k_{\mathrm{L}}^{3}}{4 \pi c \varepsilon_{0}^{2}} V_{z z}\left(k_{\mathrm{L}}, \mathbf{r}\right) \cos \left(k_{\mathrm{L}} y\right) .
$$

Here $\alpha(\omega)$ is the isotropic, dynamic, polarizability of the atoms at frequency $\omega=c k_{\mathrm{L}}=$ $2 \pi c / \lambda_{\mathrm{L}}$. The pre-factor can be expressed in terms of the Rayleigh scattering rate, $\gamma_{\mathrm{R}}$, as $I \alpha^{2} k_{\mathrm{L}}^{3} /\left(4 \pi c \varepsilon_{0}^{2}\right)=(3 / 2) \hbar \gamma_{\mathrm{R}} . \quad V_{z z}$ is the component of the retarded dipole-dipole interaction tensor generated by the linearly $\hat{z}$-polarized laser light

$$
\begin{array}{r}
V_{z z}=\frac{1}{k_{\mathrm{L}}^{3} r^{3}}\left[\left(1-3 \cos ^{2} \theta\right)(\right. \\
\left(\cos k_{\mathrm{L}} r+k_{\mathrm{L}} r \sin k_{\mathrm{L}} r\right) \\
\left.-\sin ^{2} \theta k_{\mathrm{L}}^{2} r^{2} \cos k_{\mathrm{L}} r\right]
\end{array}
$$

$\theta$ being the angle between the interatomic axis and the z-axis. The far-zone $\left(k_{\mathrm{L}} r \gg 1\right)$ behavior of (2) along the z-axis is proportional to $-\sin \left(k_{\mathrm{L}} r\right) /\left(k_{\mathrm{L}} r\right)^{2}$ and many atoms (400 at densities of $8 \times 10^{14}$ atoms $\left./ \mathrm{cm}^{3}\right)$ may lie within the 
characteristic interaction volume $\left(\lambda_{\mathrm{L}}^{3}\right)$ of this attractive long-range potential. As for the electron gas and charged Bose gas, mean-field (here Bogoliubov) theory applies in this high density regime 12 .

The laser (dynamically) induced dipole-dipole potential is distinguished from the static field $\left(r^{-3}\right)$ case 13, 14 by a longer range and a huge enhancement of atomic polarizability around a resonance. For example, in [5] ${ }^{87} \mathrm{Rb}$ atoms are magnetically trapped in the maximally stretched $\left|5 s{ }^{2} S_{1 / 2}, F=2, M=2\right\rangle$ state. A laser polarized along $\hat{z}$ is then $\pi$-polarized and only $\Delta M=0$ dipole transitions are allowed. If the light is detuned by, say, $\delta=2 \pi \times(6.5 \mathrm{GHz})$ (i.e. 1134 natural line widths) below the D1 line (795.0nm) then only virtual transitions to the $\left|5 p^{2} P_{1 / 2}, F=2, M=2\right\rangle$ state need be considered. We calculate $\alpha \approx 5.0 \times 10^{-35} \mathrm{Cm}^{2} / \mathrm{V}(\mathrm{cf}$. the static value $\left.5.3 \times 10^{-39} \mathrm{Cm}^{2} / \mathrm{V}\right)$.

In terms of the condensate density $n(\mathbf{r})$ at zero temperature, we account for atom-atom interactions using a mean-field energy functional of the form $H_{\mathrm{dd}}+H_{\mathrm{s}}$, where $H_{\mathrm{dd}}=$ $(1 / 2) \int n(\mathbf{r}) U_{\mathrm{dd}}\left(\mathbf{r}-\mathbf{r}^{\prime}\right) n\left(\mathbf{r}^{\prime}\right) d^{3} r d^{3} r^{\prime}$, and $H_{\mathrm{s}}=$ $(1 / 2)\left(4 \pi a \hbar^{2} / m\right) \int n(\mathbf{r})^{2} d^{3} r$ is due to shortrange interactions, which are described, as is usual, by a delta function pseudo-potential (we take here the repulsive case for which $a>0$ ). By working with the bare dipole-dipole interaction we assume the Born approximation also for atom-atom scattering by this long-range part of the total potential. We note that the shortrange (static) part of the laser-induced dipoledipole interaction can cause a shift in $a$. For the laser intensities and detunings considered here this shift is small according to existing estimates 14.

In a radially tight trap it is reasonable to assume a cylindrically symmetric ansatz for the density profile of radial width $w_{r}: n(\mathbf{r}) \equiv N$ $\left(\pi w_{r}^{2}\right)^{-1} n^{z}(z) \exp \left[-\left(x^{2}+y^{2}\right) / w_{r}^{2}\right]$, where $\mathrm{N}$ is the total number of atoms and $n^{z}(z)$ is normalized to 1 and left general. Denoting the FT of the atomic density by $\tilde{n}(\mathbf{k})=\int \exp [-\mathbf{i k}$. $\mathbf{r}] n(\mathbf{r}) d^{3} r$, then we have $H_{\mathrm{dd}}=(1 / 2)(2 \pi)^{-3}$ $\int \widetilde{U}_{\mathrm{dd}}(\mathbf{k}) \tilde{n}(\mathbf{k}) \tilde{n}(-\mathbf{k}) d^{3} k$, where the FT of the dipole-dipole potential (2), $\widetilde{U}_{\mathrm{dd}}(\mathbf{k})=\int \exp [-\mathbf{i k}$. $\mathbf{r}] U_{\mathrm{dd}}(\mathbf{r}) d^{3} r$, is the real part of

$$
\begin{aligned}
\widetilde{U}_{\mathrm{dd}}(\mathbf{k}) & =\frac{\mathrm{I} \alpha^{2}}{2 \epsilon_{0}^{2} c}\left(\frac{k_{z}^{2}-k_{\mathrm{L}}^{2}}{k_{x}^{2}+\left(k_{y}-k_{\mathrm{L}}\right)^{2}+k_{z}^{2}-k_{\mathrm{L}}^{2}-\mathrm{i} \eta}\right. \\
& \left.+\frac{k_{z}^{2}-k_{\mathrm{L}}^{2}}{k_{x}^{2}+\left(k_{y}+k_{\mathrm{L}}\right)^{2}+k_{z}^{2}-k_{\mathrm{L}}^{2}-\mathrm{i} \eta}-\frac{2}{3}\right) .
\end{aligned}
$$

The principal value of the radial integration in $H_{\mathrm{dd}}$ can be evaluated analytically so that the dipole-dipole energy reduces to a one dimensional functional along the axial direction $H_{\mathrm{dd}}=\left(N^{2} / 2\right) \int n^{z}(z) n^{z}\left(z^{\prime}\right) U_{\mathrm{dd}}^{z}\left(z-z^{\prime}\right) d z d z^{\prime}$ $=\left(N^{2} / 4 \pi\right) \int \widetilde{n^{z}}\left(k_{z}\right) \widetilde{n^{z}}\left(-k_{z}\right) \widetilde{U_{\mathrm{dd}}^{z}}\left(k_{z}\right) d k_{z}$, where $\widetilde{n^{z}}\left(k_{z}\right)$ is the FT of the axial density $n^{z}(z)$. The one-dimensional (1D) axial potential that appears in this expression has the form

$$
\begin{gathered}
\widetilde{U_{\mathrm{dd}}^{z}}\left(k_{z}\right)=\frac{\mathrm{I} \alpha^{2} k_{\mathrm{L}}^{2}}{4 \pi \epsilon_{0}^{2} c} Q\left(w_{r}, k_{z}\right) \\
Q\left(w_{r}, k_{z}\right)=-\frac{2}{3} \frac{1}{k_{\mathrm{L}}^{2} w_{r}^{2}}+\frac{k_{z}^{2}-k_{\mathrm{L}}^{2}}{k_{\mathrm{L}}^{2}} \mathrm{e}^{\left(k_{z}^{2}-2 k_{\mathrm{L}}^{2}\right) w_{r}^{2} / 2} \times \\
\sum_{j=0}^{\infty} \frac{\left(k_{\mathrm{L}} w_{r}\right)^{2 j}}{2^{j} j !} \Re\left\{E_{j+1}\left[\frac{\left(k_{z}^{2}-k_{\mathrm{L}}^{2}\right) w_{r}^{2}}{2}\right]\right\}
\end{gathered}
$$

where $\Re\left\{E_{j}[z]\right\}$ is the real part of the generalized exponential integral [15. The FT of the total (swave plus dipole-dipole) $1 \mathrm{D}$ reduced interatomic potential is

$$
\widetilde{U_{\text {tot }}^{z}}\left(k_{z}\right)=4 E_{\mathrm{R}} a\left(\left(k_{\mathrm{L}} w_{r}\right)^{-2}+\mathcal{I} Q\left(w_{r}, k_{z}\right)\right)
$$

where $E_{\mathrm{R}}=\hbar^{2} k_{\mathrm{L}}^{2} / 2 m$ is the photon recoil energy of an atom and $\mathcal{I}$ is the dimensionless 'intensity' parameter

$$
\mathcal{I}=\frac{\mathrm{I} \alpha^{2}(\omega) m}{8 \pi \epsilon_{0}^{2} c \hbar^{2} a} .
$$

It is emphasized that the radial degree of freedom is contained in (6) via the radius $w_{r}$. The coordinate space potential, $U_{\text {tot }}^{z}(z)$, is shown in Fig. 1 
We now have the essential ingredients to compute the excitation spectrum of the BEC as it is the FT of the effective interatomic interaction potential that appears in the Bogoliubov dispersion formula 3 . Since the influence of radial excitations upon the low-energy spectrum can be largely frozen out under tight radial confinement, we shall consider only axial phononic excitations and assume that the system is infinite along this $\hat{z}$ direction. In terms of the phonon momentum $p_{z}=\hbar k_{z}$, the axial Bogoliubov spectrum is (cf. Eq. (1))

$$
E_{\mathrm{B}}=\sqrt{c_{z}^{2} p_{z}^{2}+\left(p_{z}^{2} / 2 m\right)^{2}}=p_{z}^{2} /\left(2 m S\left(k_{z}\right)\right)
$$

where $c_{z}^{2}=\pi n(0) w_{r}^{2} \widetilde{U_{\text {tot }}^{z}}\left(k_{z}\right) / m . \quad n(0)$ is the central density in the cigar, so $\pi n(0) w_{r}^{2}$ is the linear density along the cigar. For the linear parts of the spectrum, $c_{z}$ can be interpreted as the speed of sound in the gas. Shining

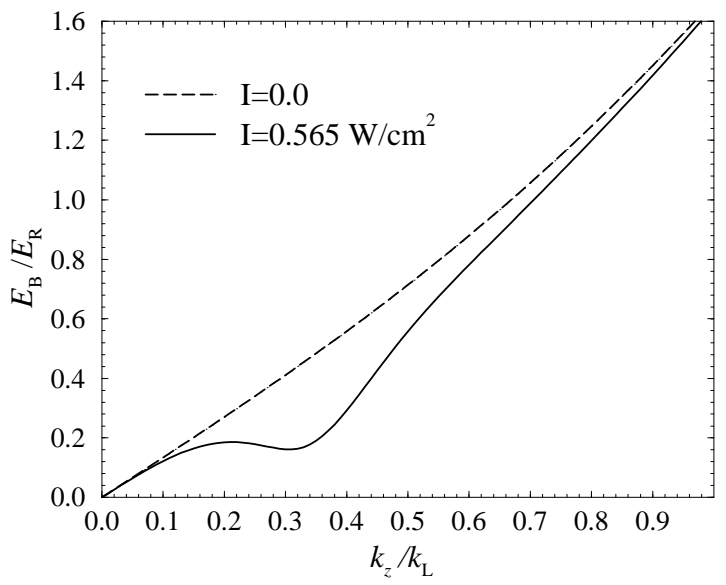

Figure 2: The Bogoliubov dispersion relation for ${ }^{87} \mathrm{Rb}$ with $w_{r}=3.5 \lambda_{\mathrm{L}}$ and $n(0)=8 \times 10^{20}$ atoms $/ \mathrm{m}^{3}$. For pure s-wave scattering $(\mathcal{I}=0)$ the inverse healing length $1 / \xi_{0}=\sqrt{8 \pi a n(0)}=$ $1.32 k_{\mathrm{L}}$.

a 795.0nm laser upon a ${ }^{87} \mathrm{Rb}$ BEC of density $n(0)=8 \times 10^{20}$ atoms $/ \mathrm{m}^{3}$ and radius $w_{r}=$ $3.5 \lambda_{\mathrm{L}}=2.78 \mu \mathrm{m}$, a 'roton' minimum appears when $\mathcal{I} \geq 0.051$ (i.e. $I \geq 0.506 \mathrm{~W} / \mathrm{cm}^{2}$ ), although the dispersion relation is considerably altered far before this. The change in the dispersion relation could be observed using Bragg spectroscopy as performed in [5]. Fig. 2] plots the Bogoliubov dispersion for $\mathcal{I}=0.057$ ( $I=$ $\left.0.565 \mathrm{~W} / \mathrm{cm}^{2}\right)$.

Local to the 'roton' minimum at $k=k_{\text {roton }}$ one can write $E=\Delta+\hbar^{2}\left(k-k_{\text {roton }}\right)^{2} / 2 m^{*}$, and for the parameters above with $\mathcal{I}=0.057$ one finds $m^{*}=0.06 m$. He II has $m^{*}=0.16 m$ 3. The static structure factor is plotted in Fig. 3. The peak in $S\left(k_{z}\right)$ corresponds to the minimum in the energy spectrum. The model described here predicts that when $\mathcal{I} \geq 0.066$ $\left(I \geq 0.654 \mathrm{~W} / \mathrm{cm}^{2}\right)$ the minimum touches the zero energy axis. At this point the system is unstable to a periodic, supersolid-like, density modulation 8, 16.

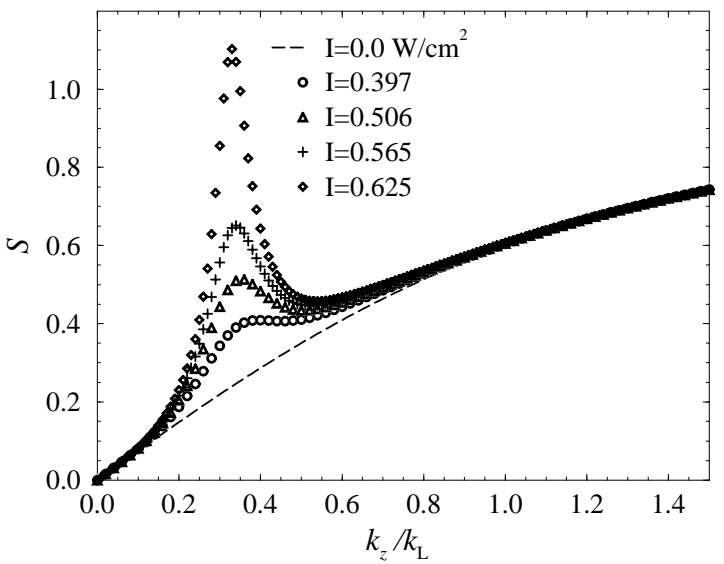

Figure 3: The static structure factor, $S\left(k_{z}\right)$ for various laser intensities. Same paramaters as Fig. [2]

The laser induced dipole-dipole potential can 
lead to electrostriction (compression) of a condensate 7]. In the present regime of low laser intensity/large detuning the electrostriction is negligible (on a scale set by the collapse threshold $\mathcal{I}=3 / 2$ [7, 13]). This regime also ensures the absence of two-body bound states in the 1D reduced potential shown in Fig. 1] a necessary condition for the validity of the Born approximation for atom-atom scattering by this potential. Only when $\mathcal{I}>1.3$ do bound states appear.

The interaction (3) arises from the forward scattering of laser photons by atom pairs. At large detunings there are two main competing processes that can heat a dense gas : A) Light-induced transfer of pairs of colliding atoms to a quasi-molecular excited state followed by dissociation, releasing $\approx \hbar \delta$ into the kinetic energy of the pair [17. This is a density dependent effect whose rate can therefore be high. Even when the laser is red-detuned from an atomic resonance, when two atoms collide the energy separation between the ground state and a molecular excited state $\left(-C_{3} / r^{3}\right)$ comes into resonance at small distances. However, by choosing $\delta$ so that the resonance point occurs between two molecular vibrational states this process is suppressed 17. Below the D1 line there are only discrete molecular vibrational states (i.e. no continuum states) so a detuning can be selected which is between these molecular resonances [18, which are narrow at ultra-cold temperatures.

B) Incoherent light scattering by single atoms occurs at approximately the Rayleigh scattering rate which can be written $\gamma_{\mathrm{R}}=$ $(8 / 3) E_{\mathrm{R}} k_{\mathrm{L}} a \mathcal{I} / \hbar$. Applying the f-sum rule for the dynamic structure factor one can show [19] that Rayleigh scattering transfers energy to the gas at a rate $\frac{d}{d t} E_{\text {tot }}=2 E_{\mathrm{R}} N \gamma_{\mathrm{R}}$ which, surprisingly, is independent of the interactions between the atoms. Comparing this heating rate with the energy of the ground state of the gas, $E_{\text {tot }} \approx H_{\mathrm{s}}+H_{\text {trap }}+H_{\text {kin }}$, where $H_{\text {kin }}$ is the kinetic energy of the atoms, one can estimate a heating time via $\tau_{\text {heat }}=E_{\text {tot }} /\left(d E_{\text {tot }} / d t\right)$. To measure a roton the BEC must survive for longer than the roton period $\tau_{\text {roton }} \propto 2 \pi \hbar / E_{R}$ (cf. Fig. 2). For the density and radius stated above, then for $\mathcal{I}=0.051$ one finds $\tau_{\text {heat }} \approx 8 \tau_{\text {roton }}$, making an experiment challenging but feasible. The situation improves for larger, denser, condensates since the polarization increases as $N^{2}$, whereas $\gamma_{\mathrm{R}}$ is a single atom effect. Finally, we note that reducing the s-wave scattering length via a Feshbach resonance allows the laser intensity (and hence the Rayleigh scattering) to be reduced by an equal factor-see Eq. (7) - and still obtain the same effects.

In conclusion, atom-atom correlations due to laser induced dipole-dipole interactions in a gaseous condensate can give a roton minimum in the Bogoliubov dispersion relation. The correlations are tunable via parameters such as radial width, laser intensity and wavelength. We thank M. Boshier, C. Eberlein, J. Steinhauer, R. Shiell, and H.T.C. Stoof for illuminating discussions, and the Engineering and Physical Sciences Research Council (EPSRC), the GermanIsraeli Foundation (GIF), and the EU QUACS and $\mathrm{CQG}$ networks for funding.

\section{References}

[1] E. Feenberg, Theory of Quantum Fluids (Academic Press, New York, 1969).

[2] K. Huang, Statistical Mechanics (Wiley, New York, 1987).

[3] E.M. Lifshitz and L.P. Pitaevskii, Statistical Physics Part 2 (ButterworthHeinemann, Oxford, 1998).

[4] www.nobel.se/physics/laureates/index.html

[5] J. Steinhauer, R. Ozeri, N. Katz and N. Davidson, Phys. Rev. Lett. 88, 120407 (2002).

[6] S. Inouye et al, Nature 392, 151 (1998).

[7] D. O'Dell, S. Giovanazzi, G. Kurizki and V.M. Akulin, Phys. Rev. Lett. 84, 5687 
(2000); S. Giovanazzi, D. O’Dell, and G. Kurizki, Phys. Rev. A 63, 031603 (2001); S. Giovanazzi, D. O'Dell, and G. Kurizki, J. Phys. B 24, 4757 (2001); S. Giovanazzi, G. Kurizki, I. E. Mazets, and S. Stringari, Europhys. Lett. 56, 1 (2001).

[8] S. Giovanazzi, D. O'Dell, and G. Kurizki, Phys. Rev. Lett. 88, 130402 (2002).

[9] S. Inouye et al, Science 285, 571 (1999); M.G. Moore and P. Meystre, Phys. Rev. Lett., 83, 5202 (1999).

[10] N. Piovella, R. Bonifacio, B.W.J. McNeil, and G.R.M. Robb, Opt. Commun. 187, 165 (2001).

[11] T. Thirunamachandran, Mol. Phys. 40, 393 (1980); D.P. Craig and T. Thirunamachandran, Molecular Quantum Electrodynamics (Academic Press, London, 1984).

[12] The validity of mean-field theory in the presence of both short-range s-wave s cattering and long-range dipole-dipole forces has been discussed in 7. See also L.L. Foldy, Phys. Rev. 124, 649 (1961).

[13] K. Góral, K. Rzążewski and T. Pfau, Phys. Rev. A 61, 051601 (2000); L. Santos, G.V. Shlyapnikov, P. Zoller and M. Lewenstein, Phys. Rev. Lett. 85, 1791 (2000).

[14] S. Yi and L. You, Phys. Rev. A 63, 053607 (2001). Fig. 1 shows shape resonances in ${ }^{41} \mathrm{~K}$ for static electric fields of $E>15 \times 10^{5}$ $\mathrm{V} / \mathrm{cm}$. The relevant quantity to compare with the present case is the atom's response $P=\alpha E$. See also P.O. Fedichev et al, Phys. Rev. Lett. 77, 2913 (1996).

[15] M. Abramowitz and I. Stegun, Handbook of Mathematical Functions (National Bureau of Standards, Washington, 1964).

[16] L.P. Pitaevskii, Zh. Eksp. Teor. Fiz. 39, 423 (1984).
[17] K. Burnett, P.S. Julienne and K.-A. Suominen, Phys. Rev. Lett. 77, 1416 (1996).

[18] J.D. Miller, R.A. Cline and D.J. Heinzen, Phys. Rev. Lett. 71, 2204 (1993).

[19] D. Pines and P. Nozières, The Theory of Quantum Liquids (Benjamin, New York, 1966) . 